\title{
Mitofusin2 Mutations Disrupt Axonal Mitochondrial Positioning and Promote Axon Degeneration
}

\author{
Albert L. Misko, ${ }^{1}$ Yo Sasaki, ${ }^{2}$ Elizabeth Tuck, ${ }^{1}$ Jeffrey Milbrandt, ${ }^{2,3}$ and Robert H. Baloh ${ }^{1,3,4}$ \\ Departments of ${ }^{1}$ Neurology and ${ }^{2}$ Genetics, and ${ }^{3}$ Hope Center for Neurological Diseases, Washington University School of Medicine, St Louis, Missouri \\ 63110, and ${ }^{4}$ Department of Neurology, Regenerative Medicine Institute, Cedars-Sinai Medical Center, Los Angeles, California 90048
}

\begin{abstract}
Alterations in mitochondrial dynamics (fission, fusion, and movement) are implicated in many neurodegenerative diseases, from rare genetic disorders such as Charcot-Marie-Tooth disease, to common conditions including Alzheimer's disease. However, the relationship between altered mitochondrial dynamics and neurodegeneration is incompletely understood. Here we show that disease associated MFN2 proteins suppressed both mitochondrial fusion and transport, and produced classic features of segmental axonal degeneration without cell body death, including neurofilament filled swellings, loss of calcium homeostasis, and accumulation of reactive oxygen species. By contrast, depletion of Opal suppressed mitochondrial fusion while sparing transport, and did not induce axonal degeneration. Axon degeneration induced by mutant MFN2 proteins correlated with the disruption of the proper mitochondrial positioning within axons, rather than loss of overall mitochondrial movement, or global mitochondrial dysfunction. We also found that augmenting expression of MFN1 rescued the axonal degeneration caused by MFN2 mutants, suggesting a possible therapeutic strategy for CharcotMarie-Tooth disease. These experiments provide evidence that the ability of mitochondria to sense energy requirements and localize properly within axons is key to maintaining axonal integrity, and may be a common pathway by which disruptions in axonal transport contribute to neurodegeneration.
\end{abstract}

\section{Introduction}

Recognizing that axons employ a non-apoptotic program of selfdestruction has been a fundamental advance in our understanding of neurodegeneration, and suggests that the molecular pathways involved could be targeted for therapeutic intervention. Evidence supports that axonal degeneration precedes cell body death in amyotrophic lateral sclerosis (Fischer and Glass, 2007), Parkinson's disease (Cheng et al., 2010), Alzheimer's disease (Vickers et al., 2000) and is a key factor determining morbidity in multiple sclerosis (MS) and other demyelinating diseases (Dziedzic et al., 2010). Therefore defining the mechanisms underlying axonal degeneration may be critical to preventing neurodegeneration.

Because of high-energy requirements and low intrinsic energy reserves, neurons require continual delivery of glucose and oxygen. Within minutes of onset, anoxia in optic nerve causes conduction block and eventually degeneration of axons (Stys et al., 1990; Malek et al., 2005). A similar scenario of "virtual hypoxia"

Received Dec. 20, 2011; revised Jan. 27, 2012; accepted Feb. 3, 2012.

Author contributions: A.L.M. and R.H.B. designed research; A.L.M., Y.S., E.T., and R.H.B. performed research; J.M. contributed unpublished reagents/analytic tools; A.L.M., E.T., and R.H.B. analyzed data; A.L.M. and R.H.B. wrote the paper.

This work was supported by National Institutes of Health Grants K08 NS055980 and R01 NS069669 (R.H.B), and AG013730 (J.M.); the Neuroscience Blueprint Core Grant NS057105 to Washington University; the Hope Center for Neurological Disorders; the Ministry of Health, Labour, and Welfare of Japan (SHA4431); Muscular Dystrophy Association Grants 4152 (R.H.B) and 10040 (J.M.); and the Children's Discovery Institute. R.H.B. holds a Career Award for Medical Scientists from the Burroughs Wellcome Fund. We thank Shaughn Bell for technical support and Sherry Clark for assistance with mouse husbandry.

Correspondence should be addressed to Dr. Robert H. Baloh at his current address: Department of Neurology, Regenerative Medicine Institute, Cedars-Sinai Medical Center, Los Angeles, CA 90048. E-mail: robert.baloh@csmc.edu.

DOI:10.1523/JNEUROSCI.6338-11.2012

Copyright $\odot 2012$ the authors $\quad 0270-6474 / 12 / 324145-11 \$ 15.00 / 0$ instigates axonal degeneration in MS where increases in nitric oxide (NO) levels inhibit mitochondrial respiration (Trapp and Nave, 2008; Trapp and Stys, 2009). In peripheral nerve explants, a transient local application of a mitochondrial membrane decoupler inhibits mitochondrial respiration at the site of exposure, and triggers Wallerian degeneration of the distal axon (Alvarez et al., 2008). Axons are thus dependent on mitochondrial production of ATP, and even a brief failure to meet energy demands anywhere along the axon leads to the degeneration of the entire distal segment.

Mitochondria are actively localized to areas of high-energy demand including the distal portion of the initial segment (Li et al., 2004), nodes of Ranvier (Berthold et al., 1993), and presynaptic boutons (Misgeld et al., 2007). These areas also experience high $\mathrm{Ca}^{2+}$ flux, which mitochondria help regulate by taking up cytosolic $\mathrm{Ca}^{2+}$ through the mitochondrial $\mathrm{Ca}^{2+}$ uniporter (Murchison and Griffith, 2007). Both ADP and $\mathrm{Ca}^{2+}$ levels can regulate mitochondrial localization (Mironov, 2007; Macaskill et al., 2009; Wang and Schwarz, 2009). Though the continual redistribution of mitochondria to maintain axonal integrity is logically implicit, it has not been experimentally demonstrated that disruption of this system compromises axonal stability.

Mutations in Mitofusin 2 (MFN2) cause axonal CharcotMarie-Tooth disease, an inherited syndrome of degeneration of long peripheral axons (Züchner et al., 2004). The mutations disrupt fusion (Detmer and Chan, 2007) and transport (Baloh et al., 2007; Misko et al., 2010) of mitochondria, and also compromise ER-mitochondrial interactions (de Brito and Scorrano, 2008). However, how these alterations in mitochondrial dynamics produce axonal degeneration has not been defined. 

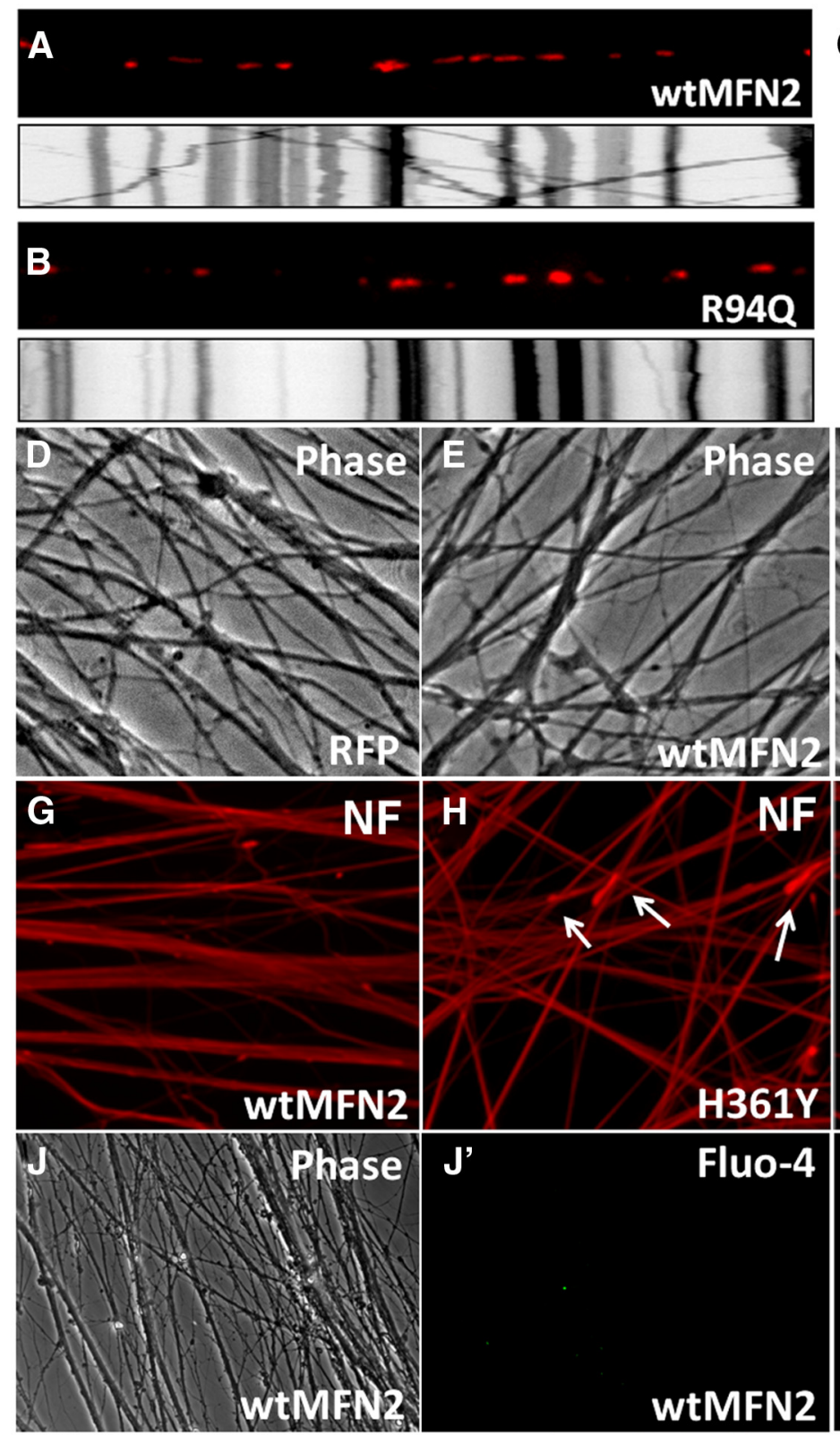

\section{J'}
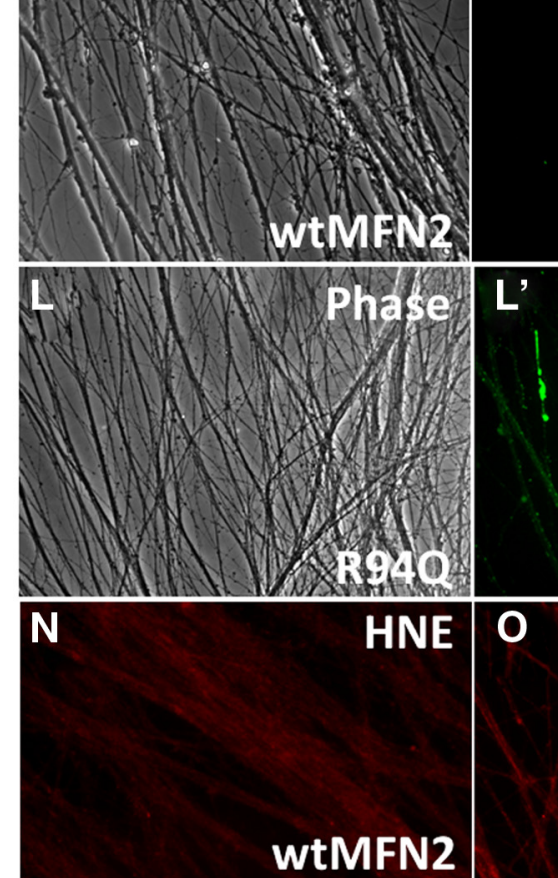

\section{L'}

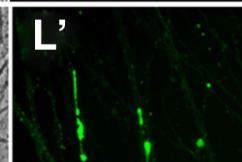

wtMFN2

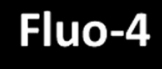

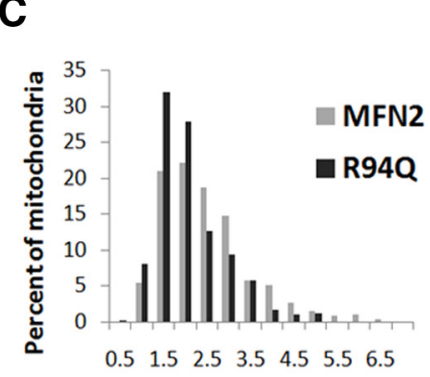

Size $(\mu \mathrm{m})$
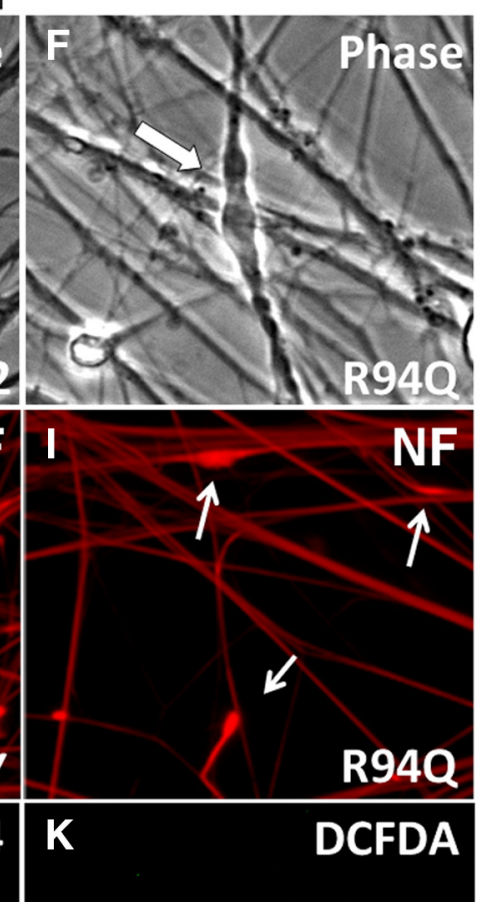

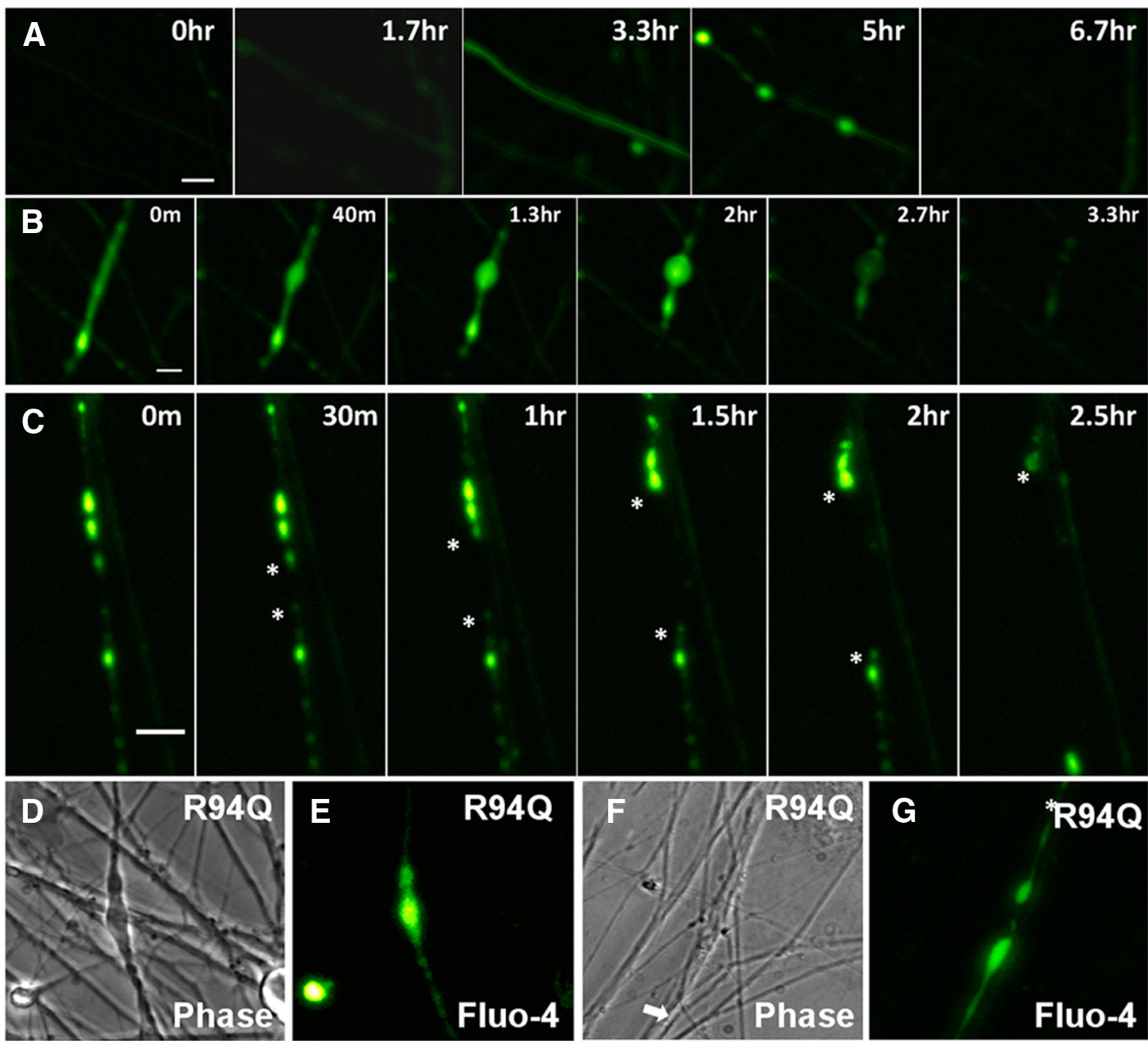

Figure 2. Axon degeneration caused by MFN2 mutant expression is segmental and dynamic. A-C, Fluo-4 imaging of MFN2 mutant-expressing axons ( $7 \mathrm{~d}$ after infection) visualized by time-lapse microscopy ( $40 \times$ magnification). Axons demonstrated a rapid (minutes) elevation in intra-axonal $\mathrm{Ca}^{2+}$, followed by beading of the membrane and finally disappearing, presumably from the inability to retain dye following loss of membrane integrity. $\boldsymbol{B}$, These frequently appeared as short segments $(\sim 50-100 \mu \mathrm{m})$ which swelled to a spheroid like shape before completely degenerating. C, Axons often lost structural integrity at a single point resulting in retraction of then ends from the break point (asterisks label ends of break point). D-G, Corresponding phase contrast and Fluo-4 images illustrate degenerating axon segments.

In this study, we investigated the relationship between altered mitochondrial dynamics and axonal degeneration using cultured neurons. We found that expression of MFN2 disease mutants produced axonal degeneration without cell body death, as in patients with MFN2 mutations. By manipulating mitochondrial fusion or transport individually, we found that axonal degeneration correlated with loss of proper mitochondrial distribution in

$\leftarrow$

(Figure legend continued.) mitochondrial mobility in R94Q-expressing neurons (number of mitochondria/min: wtMFN2, $2.03 \pm 1.16 ; \mathrm{R} 940,0.33 \pm 0.27 ; p=0.002 ; n=10$ axons per condition). C, Size frequency histogram demonstrating shorter mitochondrial lengths in R94Qexpressing neurons, indicating disruption of mitochondrial fusion. $\boldsymbol{D}-\boldsymbol{F}$, Phase imaging revealed dilated axon segments reminiscent of degenerating axon spheroids in neurons expressing R94Q but not RFP or wtMFN2, 7-10 d postinfection. G-I, Staining with antineurofilament antibody (NF) highlighted dilated axon segments. $\boldsymbol{J}-\mathbf{0}$, Degenerating segments showed increased intra-axonal $\mathrm{Ca}^{2+}$ (Fluo-4; $\left.\boldsymbol{J}^{\prime}, \boldsymbol{L}^{\prime}\right)$ and markers of reactive oxygen species [via DCFDA dye $-\boldsymbol{K}, \boldsymbol{M}$; confirmed with hydroxynonenol (HNE) immunostaining $-\boldsymbol{N}, \mathbf{0}$ ]. $\boldsymbol{P}$, Despite ongoing axonal degeneration, propidium iodide (PI) staining showed normal numbers of cell bodies, indicating a selective effect of altered mitochondrial dynamics by MFN2 mutants on axonal maintenance. Ionomycin treatment-induced cell death is shown as a positive control for the PI staining assay. axons, but not with loss of fusion, overall movement, or global mitochondrial dysfunction. We therefore propose that MFN2 disease mutants cause axonal degeneration by disrupting the ability of mitochondria to sense and position themselves appropriately to meet local metabolic need within axons.

\section{Materials and Methods}

Plasmids and silencing RNAs. Human wild-type MFN2, MFN2 disease mutants, mouse Mfn1 and opal siRNA constructs were previously described (Misko et al., 2010). GFP-SYNPH was a gift from Dr. Zu-Hang Sheng (The National Institute of Neurological Disorders and Stroke, National Institutes of Health) and was subcloned into a lentiviral expression vector.

Dorsal root ganglion cultures. Dorsal root ganglion (DRG) cultures were obtained from embryonic rats gestational day 15.5. Dorsal root ganglia were dissected out, dissociate using trypsin/EDTA, and plated directly onto 24 -well plates coated with laminin and poly-D-lysine. Cultures were maintained in Neurobasal medium with B27 supplement (Invitrogen), $50 \mathrm{ng} / \mathrm{ml} \mathrm{NGF,} 5 \mathrm{~mm}$ glutamine, and antibiotics. Addition of 5 -fluorouracil for the first $3 \mathrm{~d}$ in culture was used as an antimitotic treatment.

Lentivirus production and infection. Lentiviruses were produced as described previously (Baloh, 2008). Briefly, HEK $293 \mathrm{~T}$ cells were plated 


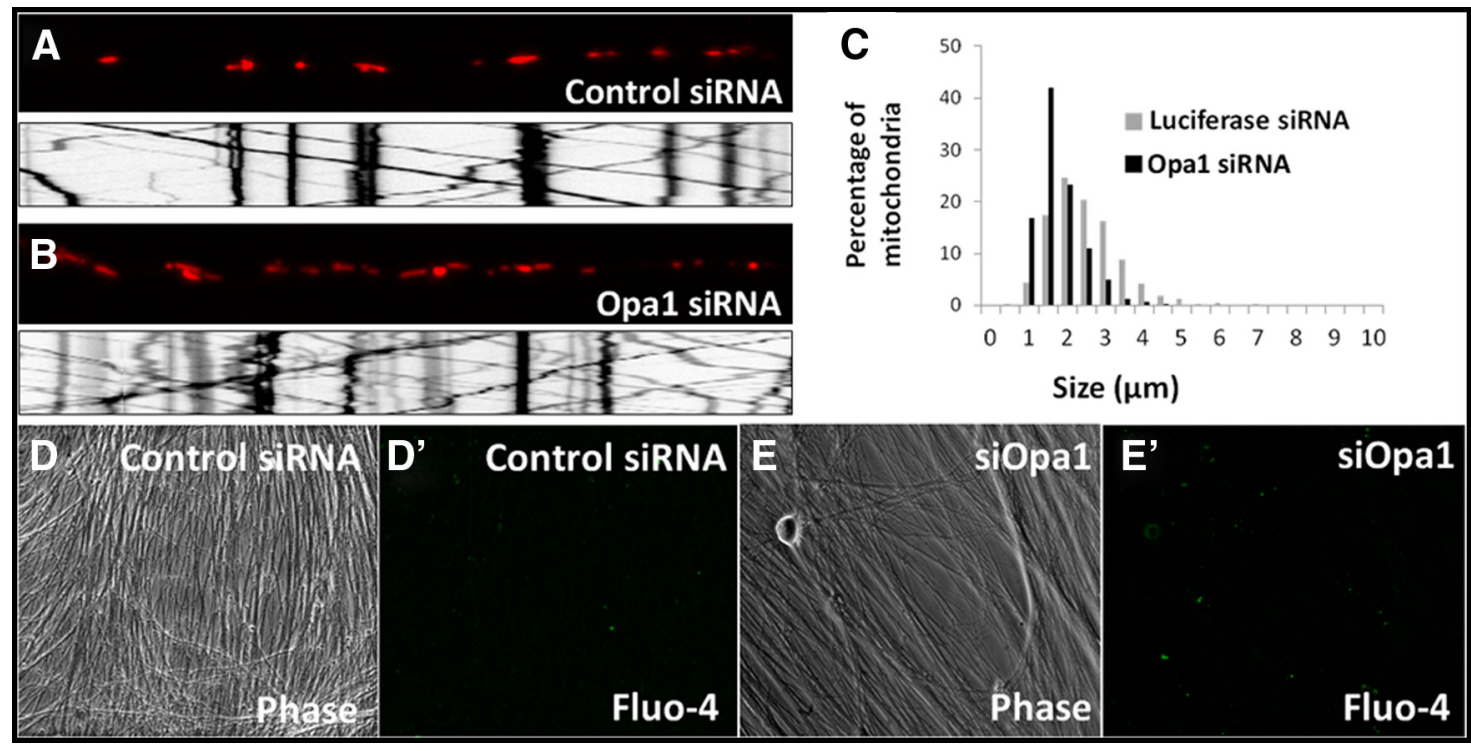

Figure 3. Disruption of mitochondrial fusion alone is not sufficient to induce axonal degeneration. DRG neurons were infected with lentivirus expressing siRNA to Opa1 (siOpa1) or luciferase (control) to inhibit mitochondrial fusion, together with mito-RFP to visualize mitochondrial dynamics via live cell imaging. $\boldsymbol{A}-\boldsymbol{C}$, Knock-down of Opa1 leaves mitochondrial transport intact ( $\boldsymbol{A}, \boldsymbol{B}$, kymographs) but results in smaller fragmented mitochondria due to diminished fusion (C). Quantification of mitochondrial flux supported normal mitochondrial mobility in siopa1-expressing neurons (number of mitochondria/min: siluc, $2.87 \pm 1.87 ;$ siopa1, 2.71 $\pm 1.51 ; p=0.828 ; n=10$ axons per condition). Unlike expression of MFN2 mutant-expressing neurons, Fluo-4 imaging detected no signs of axonal degeneration in 0 pa1 deficient neurons $\left(\boldsymbol{D}, \boldsymbol{D}^{\prime}, \boldsymbol{E}, \boldsymbol{E}^{\prime}\right)$.

onto six-well plates and transfected using Mirus reagent with a packaging vector (8.91), envelope vector (vesicular stomatitis virus-glycoprotein), and transfer vector encoding the gene to be expressed. Medium was changed once at $12 \mathrm{~h}$ and collected at $72 \mathrm{~h}$, and applied directly to DRG cultures resulting in $95 \%$ infection efficiency.

Calcium and ROS imaging. Cell permeant Fluo-4 AM (Invitrogen) was incubated at a final concentration of $1 \mu \mathrm{m}$ with cultured neurons for $30 \mathrm{~min}$ at $37^{\circ} \mathrm{C}$, washed with medium and incubated again for $30 \mathrm{~min}$ before imaging. 5- (and-6)-Carboxy-2', $7^{\prime}$-dichlorofluorescein diacetate (carboxyDCFDA) was loaded at a final concentration of $1 \mu \mathrm{M}$ for $10 \mathrm{~min}$ at $37^{\circ} \mathrm{C}$ in Neural Basal medium without B27 supplement, washed out and incubated again for $20 \mathrm{~min}$ before imaging. All microscopy including time-lapse imaging was performed with in a climate-controlled chamber (In Vivo Scientific) at $37^{\circ} \mathrm{C}$ and $5 \% \mathrm{CO}_{2}$ and images were acquired with a CoolSnap HQ $2 \mathrm{CCD}$ camera (Photometrics) mounted on a Nikon Eclipse Ti-U microscope.

Measuring mitochondrial distribution and transport. Transfection of DRG neuron cultures with mito-RFP and time-lapse imaging of mitochondrial movement were performed as previously described (Misko et al., 2010). To assess mitochondrial distribution, we adapted the protocol from (Miller and Sheetz, 2004) with minor changes. Time-lapse image stacks of axonal mitochondria within $\sim 100 \mu \mathrm{m}$ axon segments were used to create kymographs using MetaMorph software (Molecular Devices) for accurate identification of individual mitochondria. At least 10 different axons from separate neurons were imaged and divided into 10 $\mu \mathrm{m}$ bins. The number of mitochondria per bin was counted and bins were pooled for each condition. Distributions were described by comparing the average number of mitochondria $(\mu)$ per bin to the variance (var) such that $\mu>$ var is considered uniform, $\mu=$ var random and $\mu<$ var clustered. To determine significance levels we used a $\chi^{2}$ analysis of the goodness of fit of the Poisson distribution. For each condition the frequency distribution of bins with a certain number of mitochondria ( $i=0,1, \ldots 5)$ was determined $\left(f_{\mathrm{A} i}\right)$ and compared with the frequency distribution predicted by the Poisson distribution $\left(f_{\mathrm{E} i}\right)$. The predicted frequency distribution was predicted as follows:

$$
P(X)=\left(e^{-\mu}\right) \mu^{X / X !}
$$

where $P(X)$ is the probability of there being $X$ mitochondria in a bin and $\mu$ being the average number of mitochondria per bin as empirically determined for the condition being compared.

$$
f_{\mathrm{E} i}=P(X)(n)
$$

where $n$ is the total number of bins counted. The $\chi^{2}$ statistic is then calculated by comparing the observed and predicted frequency distributions.

$$
\chi^{2}=\sum_{(i=0 \text { to } i=5)}\left(f_{\mathrm{A} i}-f_{\mathrm{E} i}\right)^{2 / f_{\mathrm{E} i}}
$$

where $i$ is the number of mitochondria per bin and degrees of freedom is $d_{\mathrm{f}}=5-2=3$.

Mitochondrial flux represents the average number of mitochondria passing through the center of a bin in 1 min.

Estimating $\Delta \psi$. To measure mitochondrial membrane potentials we adopted the protocol developed by (Verburg and Hollenbeck, 2008). Briefly, DRG cultures were incubated with $20 \mathrm{~nm}$ tetramethylrhodamine methyl ester (TMRM) for $20 \mathrm{~min}$ at $37^{\circ} \mathrm{C}$, which was then exchanged for medium containing $5 \mathrm{~nm}$ TMRM in which images were acquired. A volume corrected mitochondrial to cytoplasmic fluorescence ratio $\left(F_{\mathrm{m}} / F_{\mathrm{c}}\right)$ was calculated for at least 500 mitochondria per condition by measuring the fluorescence of mitochondria and the fluorescence of the immediate surrounding axoplasm and applying the formula $F_{\mathrm{m}} / F_{\mathrm{c}}=$ $\left(\mathrm{Fl}_{\text {mitochondria }}-(2 / 3) \mathrm{Fl}_{\text {cytoplasm }}\right) /(1 / 3) \mathrm{Fl}_{\text {cytoplasm}}$.

Measuring axonal mitochondrial mass. For imaging with MitoTracker Green, cultured neurons were incubated with $75 \mathrm{~nm}$ MitoTracker Green (Invitrogen) for $30 \mathrm{~min}$ at $37^{\circ} \mathrm{C}$, washed and imaged. Labeling with TMRM is described above. To measure mitochondrial content, images of mitochondrial fluorescence were filtered with an inverted Mexican hat kernel, thresholded, and the total number of pixels representing mitochondria were counted. Phase images of corresponding areas were background subtracted and thresholded and corresponding pixels representing axons were used to normalize the mitochondrial content.

2-Deoxyglucose treatments. Medium was changed on cultures $12 \mathrm{~h}$ before 2DG administration to minimize fluctuations in metabolism from week to week. 2DG was added to cultures at a final concentration of 15 $\mathrm{mM}$ in $\mathrm{H}_{2} \mathrm{O}$ and imaged at $48 \mathrm{~h}$.

Tetrodotoxin treatment and axon degeneration measurement. DRG cultures were treated with $1 \mu \mathrm{M}$ TTX on postinfection days 4-11 following induction of mutant MFN2 constructs. Cultures were stained with DCFDA dye as described above and imaged live on postinfection day 11. To quantify axon degeneration, images of DCFDA fluorescence were 
A

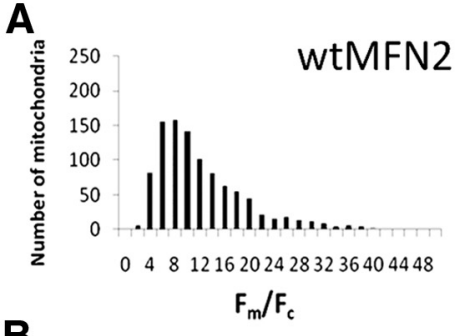

B

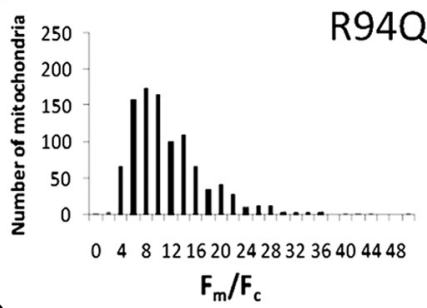

C

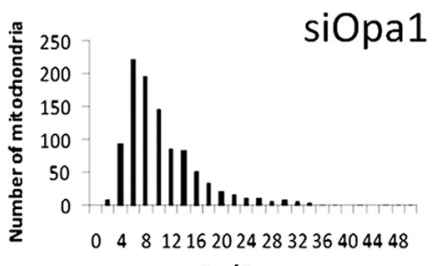

$\mathbf{F}_{\mathrm{m}} / \mathbf{F}_{\mathrm{c}}$
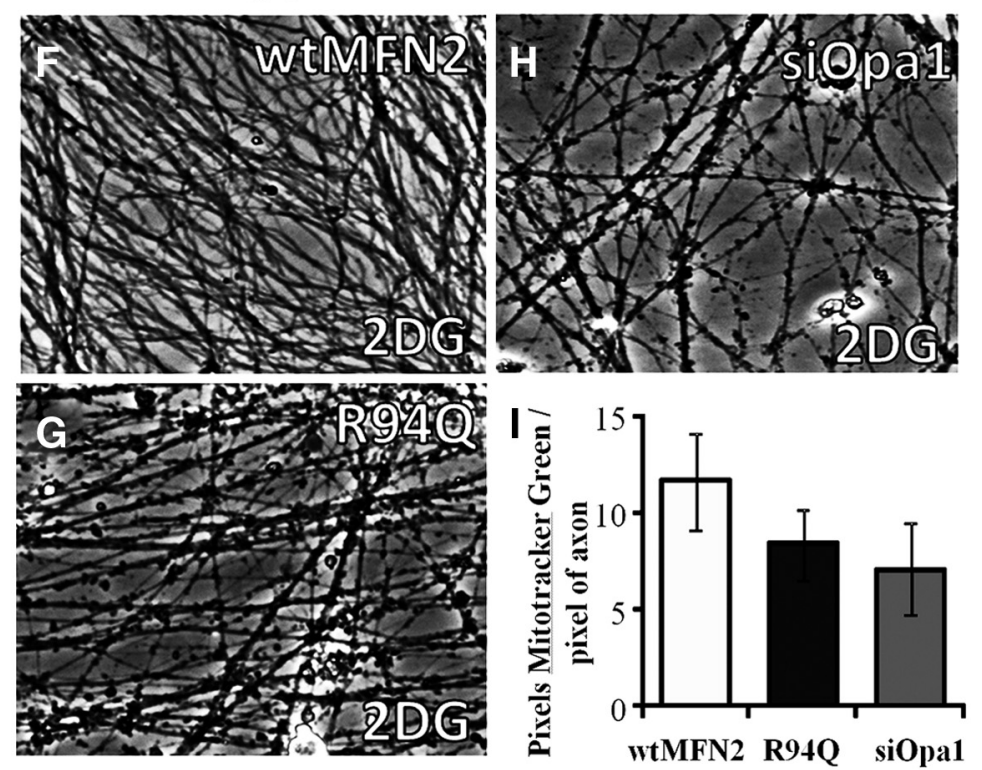

Figure 4. Mutant MFN2 and 0pa1 knockdown cause similar global suppression of mitochondrial function. $A-C$, TMRM dye was used to calculate $F_{\mathrm{m}} / F_{\mathrm{c}}$ (an estimate of $\Delta \psi$ that corrects for cytoplasmic background) values of mitochondria in wTMFN2 (A)-, R940 $(\boldsymbol{B})-$, and si0pa1 (C)-expressing cells. Mitochondria in siOpa1 showed a trend toward lower $\Delta \psi$ values, while R94Q expression did not significantly alter $\Delta \psi$ values. $\boldsymbol{D}, \boldsymbol{E}$, Despite normal levels of ATP in the cultures $(\boldsymbol{D})$, R94Q- and si0pa1-expressing cultures contained more lactate in the medium, indicating a shift toward the use of glycolysis to maintain ATP levels $(\boldsymbol{E}) . \boldsymbol{F}, \boldsymbol{G}, \boldsymbol{H}$, Treatment of cultures with $15 \mathrm{~mm} 2 \mathrm{DG}$ to inhibit glycolysis for $48 \mathrm{~h}$ produced widespread axonal degeneration (as indicated by axonal blebbing seen on phase contrast images) in R94Q- and si0pa1-expressing cultures, supporting the notion that the axons are more reliant on glycolysis to maintain ATP levels. I, MitoTracker Green staining of R94Q- and siOpa1-expressing cultures revealed an $\sim 20 \%$ and $40 \%$ decrease in mitochondrial mass, respectively.

thresholded, and the total number of pixels, representing degenerating axons, were determined. Phase images of corresponding areas were background subtracted, thresholded, and corresponding pixels representing total axons were used to normalize the measure of degenerating axons. Axonal degeneration in MFN2 mutant-expressing cultures was normalized to $100 \%$ for each mutant (R94Q and H361Y), and the decrease in axonal degeneration from TTX treatment was graphically represented as
"\% control." Therefore the control here represents the basal level of axonal seen in mutant MFN2-expressing cultures to compare to drug treatment.

\section{Results}

Expression of MFN2 disease mutants causes segmental axonal degeneration without cell body death in cultured sensory neurons

Point mutations in MFN2 that cause inherited axon degeneration in humans disrupt mitochondrial transport and also alter mitochondrial fusion (Baloh et al., 2007; Detmer and Chan, 2007; Misko et al., 2010). However the mechanism by which altered mitochondrial dynamics leads to length-dependent axon degeneration in the peripheral nervous system remains unclear. To investigate this we introduced wild-type (wtMFN2) or disease mutant MFN2 (R94Q and H361Y) into cultured DRG neurons using lentivirus, and monitored both mitochondrial dynamics and axonal stability. As expected, expression of MFN2 disease mutants altered several aspects of mitochondrial dynamics, dominantly inhibiting both mitochondrial fusion and movement within $3 \mathrm{~d}$ of expression, as in prior studies (Fig. $1 A-C$ ) (Misko et al., 2010). These changes were specific to MFN2 disease mutants, and occurred at low levels of lentiviral mediated MFN2 expression (1.2- to 1.4-fold above endogenous).

While disruption of mitochondrial transport and fusion did not produce obvious cell death, frequent scattered dilated axonal segments and beading were seen on phase contrast imaging starting at $7 \mathrm{~d}$ after infection (Fig. $1 F$ ). Immunostaining for neurofilament confirmed that numerous dilated axon segments were present in mutant (R94Q, H361Y)- but not wtMFN2-expressing cultures, consistent with ongoing axonal degeneration (Fig. $1 G-I)$. Axonal degeneration is also often accompanied by loss of $\mathrm{Ca}^{2+}$ homeostasis and accumulation of ROS (Trapp and Stys, 2009). MFN2 mutant-expressing cultures revealed scattered beaded and dilated axons intensely positive for Fluo-4 (indicating elevated $\mathrm{Ca}^{2+}$ levels) and DCFDA or HNE immunostaining (markers of ROS) (Fig. $1 J-L$ ), providing further support that altered mitochondrial dynamics produces characteristic features of axonal degeneration in cultured neurons. Features of axonal degeneration (swelling, beading; Fluo-4, DCFDA, or HNE staining) were never observed in wtMFN2expressing cultures (Fig. $\left.1 E, G, J, J^{\prime}, K, N\right)$ ). Axonal degeneration from mutant MFN2 expression was not a consequence of cell body death, as cell counting revealed a similar number of cell bodies in wtMFN2- and R94Q-expressing cultures (96.4 $\pm 3 \%$ 
and $97.2 \pm 3 \%$, respectively) after $7 \mathrm{~d}$ in culture (Abhyankar et al., 2007). Likewise, no cell death was detected using propidium iodide staining when axonal degeneration was widespread (Fig. 1M). These experiments confirmed that the observed degeneration originated in axons and was not a consequence of cell death.

To examine the dynamics of elevated intra-axonal $\mathrm{Ca}^{2+}$ in relation to axonal degeneration, we performed time lapse microscopy 7-10 d after expressing wtMFN2 or R94Q-MFN2 using Fluo-4 dye (Fig. 2). Time lapse movies were taken over $12 \mathrm{~h}$, and three patterns of degeneration were identified: (1) axons displaying a normal caliber and uniform rise in intracellular $\mathrm{Ca}^{2+}$, followed by beading and degeneration (seen as disappearance of the axon after loss of membrane integrity and inability to retain dye); (2) dilated axon segments swelling to spheroids, followed by loss of membrane integrity; (3) axons breaking apart from a single point with the ends retracting. From the onset of increased intracellular $\mathrm{Ca}^{2+}$, most axons degenerated within $\sim 3 \mathrm{~h}$, and no axons were observed to recover (i.e., did not progress to blebbing and fragmentation).

Together these data indicate disruption of mitochondrial transport and fusion from MFN2 mutant expression leads to segmental loss of $\mathrm{Ca}^{2+}$ homeostasis and axonal degeneration in cultured neurons without evidence of cell body death.

\section{Axonal degeneration does not result from loss of mitochondrial fusion or globally decreased mitochondrial function}

Decreased levels of Opa1, a dynamin family GTPase required for inner mitochondrial membrane fusion, drastically suppresses mitochondrial fusion and produces mitochondrial dysfunction (Chen et al., 2005), while leaving microtubule-based mitochondrial transport intact (Misko et al., 2010). Given that MFN2 disease mutants produce a "mixed lesion" of both mitochondrial transport and mitochondrial fusion, we asked whether disruption of mitochondrial fusion was sufficient to cause axonal degeneration. Therefore we used a siRNA against Opal (siOpa1) to selectively disrupt mitochondrial fusion without altering transport, and looked for signs of axonal degeneration. Three days after knockdown of Opa1, time-lapse microscopy confirmed normal patterns of mitochondrial transport (Fig. $3 A, B$, kymographs), however mitochondria were markedly smaller consistent with suppression of fusion caused by Opal loss (Fig. 3C). However despite marked suppression of mitochondrial fusion, imaging with Fluo-4 or DCFDA dye showed no signs of axon degeneration $7 \mathrm{~d}$ after infection (Fig. 3D-I), despite the presence of marked mitochondrial fragmentation. These results indicate that disrupting mitochondrial fusion alone was not sufficient to cause axon degeneration.
Decreased mitochondrial fusion leads to suppression of mitochondrial function, including loss of membrane potential $(\Delta \psi)$ and oxidative respiration (Chen et al., 2005). To interrogate mitochondrial function in our cultures, we used TMRM dye to calculate the $F_{\mathrm{m}} / F_{\mathrm{c}}$ value of axonal mitochondria as an estimate of $\Delta \psi$ (Verburg and Hollenbeck, 2008). One week after infection with viral constructs, mitochondria in R94Q-MFN2-expressing neurons maintained a $F_{\mathrm{m}} / F_{\mathrm{c}}$ distribution comparable to those in wtMFN2 controls (Fig. 4A,B), whereas mitochondria in siOpa1 infected neurons showed a minor shift in population distribution toward lower values of $F_{\mathrm{m}} / F_{\mathrm{c}}$. (Fig. $4 C$ ). To further assess mitochondrial function, we looked for a shift toward anaerobic metabolism by measuring lactate levels in the culture medium. Both R94Q-MFN2- and siOpa1-expressing cultures contained more lactate compared with controls, supporting a shift toward increased utilization of glycolysis to maintain normal ATP levels (Fig. 4D,E). To confirm the reliance on glycolytic production of ATP, cultures were treated with $15 \mathrm{~mm}$ 2DG to inhibit glycolysis. 
After $48 \mathrm{~h}$ of treatment with 2DG, signs of axonal degeneration were apparent in both R94Q-MFN2- and siOpa1-expressing neurons, but not wtMFN2-expressing controls. Finally, mitochondrial mass was decreased in both R94Q- and siOpal-expressing cells ( $\sim 20 \%$ and $40 \%$ respectively; Fig. $4 I$ ), presumably reflecting the autophagic removal of mitochondria with decreased membrane potential (Twig et al., 2008).

These data indicated that suppression of mitochondrial fusion, from either knockdown of Opa1 or MFN2 mutant expression, led to a loss of mitochondrial mass and global mitochondrial dysfunction in cultured neurons. However, since axonal degeneration was not observed from knockdown of Opal, mild global mitochondrial dysfunction from loss of mitochondrial fusion alone could not explain the segmental axonal degeneration observed from expression of MFN2 disease mutants. As noted above, in addition to mediating mitochondrial fusion, MFN2 is required for mitochondrial transport via direct interactions with the microtubule-based transport apparatus (Misko et al., 2010). Therefore we examined the relationship between alterations in mitochondrial transport and axonal degeneration.

\section{Loss of proper axonal mitochondrial distribution, rather than disruption of bulk transport, is correlated with axonal degeneration}

To define the relationship between altered mitochondrial movement and axonal degeneration, we overexpressed syntaphilin (Synph), an outer mitochondrial membrane protein which suppresses mitochondrial movement by anchoring mitochondria to microtubules, but does not influence mitochondrial fusion or interact with MFN2 (Kang et al., 2008; Misko et al., 2010). Overexpression of Synph markedly suppressed mitochondrial movement in DRG axons by $3 \mathrm{~d}$ after infection, similar to that observed by expression of MFN2 disease mutants (Fig. $5 A, B$ ) but did not affect mitochondrial fusion (Fig. $5 C$ ). Unlike mutant MFN2 expression however, at 7-10 d after infection Synph overexpression did not induce signs of axonal degeneration (Fig. 5D,E). This indicated that simply blocking overall mitochondrial movement in axons was insufficient to induce axonal degeneration. Of note, we observed that while overall movement was suppressed in both conditions, mitochondria in R94Q-expressing neurons frequently showed clusters with large gaps between mitochondria, which were not observed in Synph-overexpressing neurons (see below).

In axons, mitochondria distribute to areas of high energy demand where they supply ATP and buffer $\mathrm{Ca}^{2+}$, functions critical to axonal integrity (for review, see Baloh, 2008). In cultured DRG neurons, mitochondria distribute uniformly along axons, consistent with their ability to sense energy requirements and position where they are needed (Miller and Sheetz, 2004). MFN2 interacts directly with Mirol and Miro2, OMM proteins which function as $\mathrm{Ca}^{2+}$ sensors to regulate mitochondrial localization to sites of high $\mathrm{Ca}^{2+}$ (Saotome et al., 2008; Macaskill et al., 2009; Wang and Schwarz, 2009; Misko et al., 2010). Therefore we investigated whether MFN2 mutants disrupt the ability of mitochondria to sense energy demand and properly distribute in axons, which would compromise axonal integrity.

Mitochondria in DRG neurons infected with wtMFN2, R94Q-MFN2, Synph, or siOpa1 constructs were labeled with mito-RFP and imaged by time-lapse microscopy. Kymographs were used to identify stationary mitochondria, represented as black dots underneath kymographs (Fig. 6). While the mitochondrial distribution in wtMFN2-, Synph-, or siOpa1-expressing cells appeared uniform (Fig. 6A,C), axon segments in R94Q-

\section{A wtMFN2}

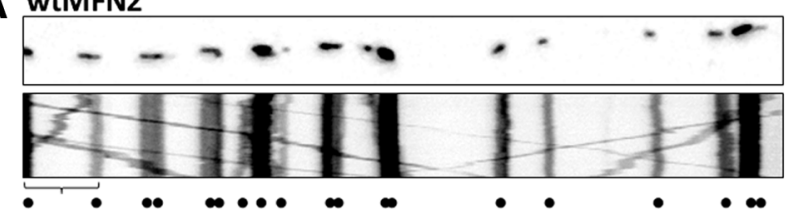

B R94Q-MFN2

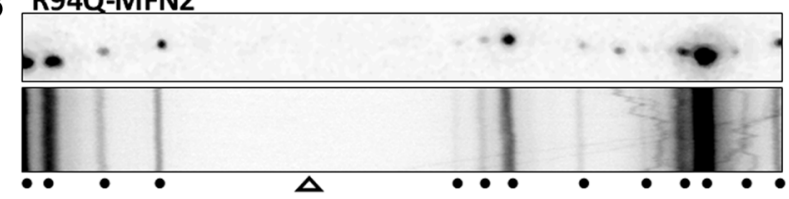

C siopa1

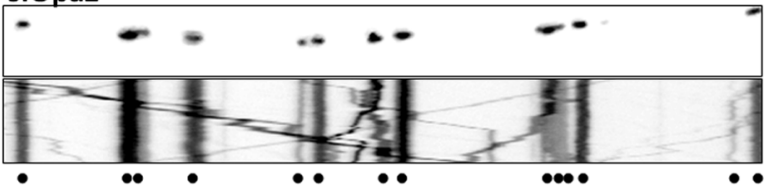

D Synph

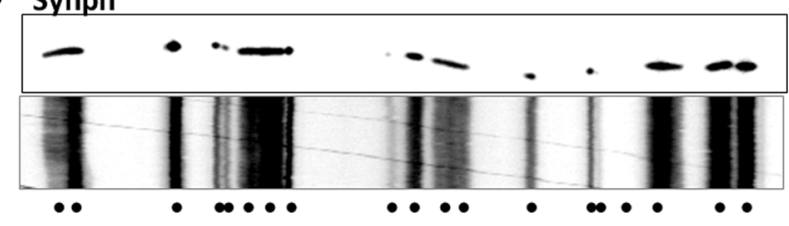

Figure 6. Abnormal distribution of axonal mitochondria correlates with axonal degeneration from mutant MFN2 expression. Mitochondria in cultured DRG neurons expressing virally transduced wtMFN2, R94Q, si0pa1 or Synph were labeled with mito-RFP and imaged by timelapse microscopy 1 week after infection. $\boldsymbol{A}-\boldsymbol{D}$, Kymographs were used to assist in the identification of fluorescently labeled mitochondria. Individual mitochondria are represented as black dots below kymographs. While mitochondria were evenly distributed along axons of wtMFN2-, Synph-, and si0pa1-expressing neurons $(\boldsymbol{A}, \boldsymbol{C}, \boldsymbol{D})$, R94Q mutant-expressing axons commonly displayed prolonged segments that were devoid of mitochondria ( $\boldsymbol{B}$, arrowhead). Axons from 10 neurons were divided into $10 \mu \mathrm{m}$ bins (length of bracket in $\mathrm{A}$ ) and the number of mitochondria counted per bin. Mitochondrial distributions were compared with a Poisson distribution with a $\chi^{2}$ goodness of fit test as described in the text and shown in Table 1.

\section{Table 1. Mitochondrial distribution in axons}

\begin{tabular}{lccrrr}
\hline & wtMFN2 & R94Q & si0pa1 & GFP & Synph \\
\hline Average number of mitochondria/bin & 2.1 & 1.52 & 1.98 & 1.24 & 1.93 \\
Variance & 1.14 & 1.52 & 1.37 & 0.41 & 0.65 \\
$c^{2}$ & 19.89 & 4.99 & 18.16 & 50.05 & 73.79 \\
p-value & $<0.001$ & $\mathbf{0 . 0 2 8 8}$ & $<0.001$ & $<0.001$ & $<0.001$
\end{tabular}

Bold $p$-value, Not significantly different from the Poisson distribution $(p<0.001)$.

expressing cells were often devoid of mitochondria (Fig. $6 B$, arrowhead). To quantitate mitochondrial distribution, we used a previously described statistical method that was used to show that mitochondria are uniformly distributed in axons of cultured DRG neurons (Miller and Sheetz, 2004). Axons were divided into $10 \mu \mathrm{m}$ bins and mitochondria were counted in at least 10 bins per axon across 10 different axons. A mitochondrial population was considered uniformly distributed if the average number of mitochondria per bin $(\mu)$ was greater than the variance (var), random if $\mu=$ var and clustered if $\mu<$ var. By this measure, mitochondria were uniformly distributed in cells expressing wtMFN2, Synph, or siOpal, but randomly distributed in R94Q-expressing cells (Table 1). Comparing observed distributions to the Poisson distribution with the same mean by using a $\chi^{2}$ goodness of fit test confirmed that mitochondrial distributions in wtMFN2, Synph, and siOpal axons differed significantly from the Poisson distribution $(p<0.001)$ (i.e., were evenly distributed) while the pop- 
ulation in R94Q-expressing neurons did not $(p=0.0288)$ (i.e., were unevenly distributed) (Table 1).

These data support that altered axonal mitochondrial distribution, rather than loss of overall mitochondrial movement per se, is responsible for axonal degeneration in mutant MFN2-expressing cultures.

\section{Sodium channel blockade, and Mfn1 overexpression can prevent axonal degeneration in MFN2 disease mutant-expressing cells}

The findings above suggest that the segmental degeneration observed in axons expressing MFN2 mutants occurs at local regions devoid of mitochondria, which are functionally "hypoxic." The cascade of molecular events perpetuating the degeneration of hypoxic axons has been well characterized (Stirling and Stys, 2010). Energy deficits in an axon lead to failure of the $\mathrm{Na}^{+}-\mathrm{K}^{+}$pump, which allows $\mathrm{Na}^{+}$to accumulate in the cytoplasm. This forces the $\mathrm{Na}^{+}-\mathrm{Ca}^{2+}$ exchanger to reverse directions and triggers an influx of $\mathrm{Ca}^{2+}$ that elicits the final stages of axon degeneration (Stys et al., 1992). In experimental hypoxia paradigms, addition of TTX to rat optic nerve obstructs influx of extracellular $\mathrm{Na}^{+}$and prevents downstream degeneration (Wolf et al., 2001). We reasoned that if the abnormal distribution of mitochondria in MFN2 mutant-expressing neurons left axonal segments devoid of mitochondria in a state of virtual hypoxia, then treatment of these cultures with TTX would prevent their degeneration. Therefore we treated mutant MFN2-expressing DRG cultures with TTX, and cultures were imaged at $7 \mathrm{~d}$ later with DCFDA dye to visualize segmental axon degeneration (Fig. $7 A-D$ ). Treatment with TTX markedly decreased the number of DCFDA-positive axons in mutant-expressing cultures (Fig. 7E). These data support that local loss of mitochondrial function leads to "virtual hypoxia" and segmental axon degeneration, and that blocking the influx of $\mathrm{Na}^{+}$via TTX can prevent the cascade of axonal degeneration similar to the protection observed in experimentally hypoxic axons.

We recently showed that increased expression of Mfn1 in cultured neurons could restore normal axonal transport of mitochondria in Mfn2-null DRG neurons, as basal levels of Mfn1 is low in these neurons relative to non-neuronal cells (Misko et al., 2010). Furthermore, another study found that expression of Mfn1 could also complement the fusion defect induced by some MFN2 disease mutants (Detmer and Chan, 2007). Therefore, to investigate augmentation of Mfn 1 expression as a potential therapeutic strategy for CMT2A, we examined whether expression of exogenous Mfn1 could prevent the axon degeneration observed in cultures expressing the R94Q MFN2 mutant. Neurons were first infected with lentivirus containing an R94Q construct, and subsequently a second lentivirus containing a Mfn 1 construct. When cultures were imaged with Fluo- 4 dye 1 week later, signs of axonal degeneration were completely absent (Fig. $8 A-D)$. Intro- duction of Mfn 1 rescued mitochondrial transport and content in axons, indicating that increased expression of Mfn1 above endogenous levels is able to overcome the dominant effect of the MFN2 R94Q mutant on mitochondrial dynamics (Fig. 8E,F). These data indicate that augmenting levels of Mfn 1 in the nervous system of CMT2A patients may offer a viable strategy for therapeutics development.

\section{Discussion}

These experiments demonstrate that MFN2 mutant proteins alter mitochondrial dynamics and trigger segmental axon degeneration in cultured neurons. Although MFN2 mutants altered both the overall movement and fusion of mitochondria, by comparison to other interventions (blocking fusion with Opal knockdown, or bulk movement with Synph overexpression), the key abnormality produced by MFN2 mutants that correlated with axonal degeneration was the alteration of proper distribution of mitochondria along axons. This suggests that MFN2 mutants disrupt the sensing mechanisms based at the outer mitochondrial membrane that organize mitochondrial position within axons, consistent with their interactions with components of the Miro- 

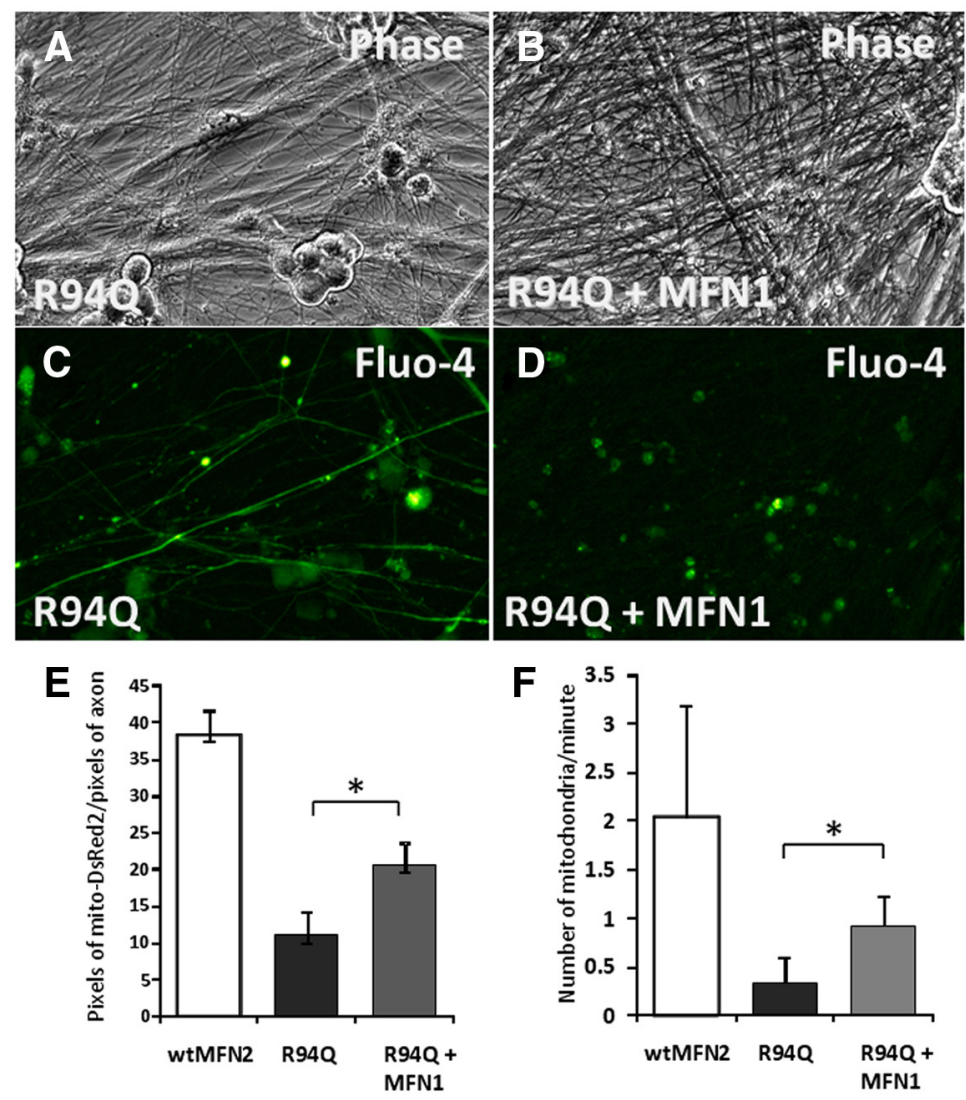

Figure 8. Expression of $M f n 1$ rescues axonal degeneration induced by MFN2 disease mutants. $A-D$, Cultured DRG neurons were infected with R94Q mutant MFN2 on DIV3, and subsequently infected with control or Mfn1 constructs on DIV4. Whereas Fluo-4 imaging showed frequent degenerating axons in cultured neurons expressing R94Q-MFN2 as before, cultures coinfected with Mfn1 and R94Q-MFN2 showed no signs of axonal degeneration $7 \mathrm{~d}$ after infection. $\boldsymbol{E}, \boldsymbol{F}$, Expression of Mfn1 rescued mitochondrial transport, as measured both by the amount of mitochondria migrating into axons after infection with mito-DsRed2 virus $\left(\boldsymbol{E}^{*}{ }^{*} p<\right.$ 0.001 , Student's $t$ test), and frequency of mitochondrial movement measured as mitochondria/per minute crossing an arbitrary point along the axon $\left(\boldsymbol{F} ;{ }^{*} p<0.001\right.$, Student's $t$ test $)$.

Milton-Kinesin system. This segmental "virtual hypoxia" as the driver of degeneration in mutant MFN2-expressing cells was supported by the ability of TTX to prevent axonal degeneration, similar to other models of hypoxic axonal injury. These data therefore provide a direct link between altered mitochondrial dynamics and axonal degeneration, and specifically point to disruption of local axonal mitochondrial energy sensing and positioning as a key factor. As altered axonal transport is a common feature of most neurodegenerative and demyelinating diseases, the disruption of local mitochondrial distribution within axons may be a key step leading to axon degeneration in a diverse set of diseases beyond CMT2A.

\section{Mitochondrial function in the maintenance of axon integrity} The conduction of action potentials along nerves and white matter tracks is costly, requiring energy to pump ions across the plasma membrane and establish the resting membrane potential. Despite the high demands of action potential propagation, neurons have low intrinsic energy reservoirs and require a continuous supply of oxygen and glucose. Disruption of energy production in axons initiates a series of events leading to axonal degeneration: (1) failure of the $\mathrm{Na}^{+}-\mathrm{K}^{+}$pump from lack of ATP; (2) $\mathrm{Na}^{+}$accumulation through persistent $\mathrm{Na}^{+}$channels and $\mathrm{K}^{+}$ efflux; and (3) reversal of the $\mathrm{Na}^{+}-\mathrm{Ca}^{2+}$ exchanger driven by high axoplasmic $\mathrm{Na}^{+}$and consequent accumulation of intracellular $\mathrm{Ca}^{2+}$ (Stys et al., 1992). High intra-axonal $\mathrm{Ca}^{2+}$ marks a late stage in axon degeneration leading to the activation of calpains, and degradation of the neurofilament network (Trapp and Nave, 2008). Even a transient local deficit in mitochondrial metabolism can be sufficient to trigger axonal degeneration. Experimental exposure of peripheral nerves to NO, which inhibits mitochondrial respiration, initiates Wallerian degeneration that spreads distal to the administration site, even when the NO is removed within $3 \mathrm{~h}$ of exposure. The same effects are seen with a brief administration of DNP, an uncoupler of mitochondrial respiration (Alvarez et al., 2008). Similar local deficits are likely to arise when disruption of mitochondrial transport leaves segments of axon devoid of mitochondria, creating a state of local "virtual hypoxia." Our data suggest that this mechanism may be the inciting cause of axon degeneration in CMT2A.

The importance of mitochondrial energy sensing and proper movement is also relevant to other neurodegenerative diseases. In demyelinating disorders such as MS, mitochondria accumulate at denuded axon segments, presumably to meet the increased energy demands of action potential propagation (Andrews et al., 2006; Hogan et al., 2009; Witte et al., 2009). In damaged axons in brains of humans with multiple sclerosis and of mice with cuprizone-induced demyelination, elevated levels of cytosolic HDAC1 were shown to directly inhibit mitochondrial transport (Kim et al., 2010). The increased need for mitochondria at denuded axon segments suggests that secondary axonal injury would be increased if mitochondrial transport was disrupted. Developing drugs to enhance the efficacy of proper mitochondrial transport and localization may improve the survival of demyelinated axons by increasing their mitochondrial reserves.

Disrupted mitochondrial transport has also been implicated in numerous neurodegenerative diseases including Alzheimer's disease, Huntington's disease, amyotrophic lateral sclerosis and others (Morfini et al., 2009). Though the mechanism by which axonal transport is disrupted is likely different in the various neurodegenerative diseases, altered mitochondrial transport and localization may be a key and early event, which drives axonal degeneration similar to that observed from expression of MFN2 disease mutants.

\section{The connection between segmental energy crisis and} length-dependent axon degeneration

A striking feature of CMT2A is the predilection for degeneration of peripheral sensory and motor neurons with long axons, despite the ubiquitous expression of MFN2 in all cells. It is reasonable to consider that long axons may be more susceptible to deficits in mitochondrial transport based on their length for several reasons. First, abnormal transport could significantly delay or even prevent the migration of newly synthesized mitochondria in the cell body from reaching the most distal axon segments. However it is 
notable from several studies of patient biopsies that diminished mitochondrial populations are not evident in sural nerves from CMT2A patients, and instead clusters of small mitochondrial are frequently seen in distal axons (Verhoeven et al., 2006; Vallat et al., 2008). There is also evidence that mitochondrial biogenesis may take place in axons negating the dependency on transport of mitochondria from the soma (Amiri and Hollenbeck, 2008; Vincent et al., 2010). The data shown here support that a deficit in regulation of local (rather than long range) transport and distribution of mitochondria to regions of energy demand along axons would compromise axon integrity and drive axonal degeneration. Assuming random fluctuations in ATP and $\mathrm{Ca}^{2+}$ levels occur at some frequency per a given length of axon, then longer axons would encounter these events more frequently. Therefore an overall compromise in the ability of mitochondria to move to these sites and restore $\mathrm{Ca}^{2+}$ homeostasis would still lead to preferential degeneration of the longest axons. Indeed, biopsies from patients with MFN2 mutations have frequently shown clustering of small round mitochondria within axons, consistent with the observed alterations in mitochondrial distribution observed in our culture system (Verhoeven et al., 2006; Vallat et al., 2008; Calvo et al., 2009).

Another potential connection between MFN2 mutants and intra-axonal $\mathrm{Ca}^{2+}$ homeostasis would be the role for MFN2 as a tether between mitochondria and the ER. de Brito and Scorrano (2008) reported that ER morphology was abnormal in Mfn2 ${ }^{-1-}$ MEFs lacking endogenous Mfn2, and that R94Q mutant MFN2 was not able to rescue the ER defect. Here we modeled the effects of R94Q mutant MFN2 in cells with normal levels of endogenous Mfn2, to mimic the dominant effect of these mutants seen in CMT2A patients. Unfortunately we were not able to obtain sufficient material from DRG neurons to directly measure ERmitochondrial tethering biochemically. However, since Mfn1 expression was able to rescue $\mathrm{Ca}^{2+}$ homeostasis and axonal degeneration induced by MFN2 mutants, this argues that in our culture system MFN2 mutants do not promote axonal degeneration by disrupting ER-mitochondrial tethering and $\mathrm{Ca}^{2+}$ buffering, because Mfn1 does not rescue ER-mitochondrial tethering (de Brito and Scorrano, 2008). By contrast, Mfn1 is able to rescue the mitochondrial transport abnormalities seen in $\mathrm{Mfn} 2^{-1-}$ DRG neurons (Misko et al., 2010), supporting it is the recovery of normal mitochondrial transport and localization that mediates the protective effect of Mfn1 overexpression in MFN2 mutantexpressing neurons.

\section{Modulating Mfn1 expression as a neuroprotective strategy in the treatment of CMT2A}

It is also possible that the lower level of Mfn1 expression relative to other cell types contributes to the sensitivity of peripheral neurons to MFN2 mutations (Detmer and Chan, 2007), in addition to their requirement for carefully regulated mitochondrial transport. We previously reported that levels of Mfn 1 protein in DRG neurons were somewhat lower than mouse embryonic fibroblasts, and observed a different migration pattern on Western blotting (Misko et al., 2010). Furthermore we found here that augmenting Mfn1 levels via lentiviral expression was able to rescue faulty axonal transport and the axonal degeneration produced by MFN2 mutant expression. Though it is hard to know what levels of Mfn1 would be required to provide a similar compensatory recovery in patients, efforts toward understanding transcriptional and translational regulation of Mfn1 in neurons to augment its expression could be a useful strategy toward developing therapeutic agents for CMT2A.

\section{References}

Abhyankar MM, Urekar C, Reddi PP (2007) A novel CpG-free vertebrate insulator silences the testis-specific SP-10 gene in somatic tissues: role for TDP-43 in insulator function. J Biol Chem 282:36143-36154.

Alvarez S, Moldovan M, Krarup C (2008) Acute energy restriction triggers Wallerian degeneration in mouse. Exp Neurol 212:166-178.

Amiri M, Hollenbeck PJ (2008) Mitochondrial biogenesis in the axons of vertebrate peripheral neurons. Dev Neurobiol 68:1348-1361.

Andrews H, White K, Thomson C, Edgar J, Bates D, Griffiths I, Turnbull D, Nichols P (2006) Increased axonal mitochondrial activity as an adaptation to myelin deficiency in the Shiverer mouse. J Neurosci Res 83:1533-1539.

Baloh RH (2008) Mitochondrial dynamics and peripheral neuropathy. Neuroscientist 14:12-18.

Baloh RH, Schmidt RE, Pestronk A, Milbrandt J (2007) Altered axonal mitochondrial transport in the pathogenesis of Charcot-Marie-Tooth disease from mitofusin 2 mutations. J Neurosci 27:422-430.

Berthold CH, Fabricius C, Rydmark M, Andersén B (1993) Axoplasmic organelles at nodes of Ranvier. I. Occurrence and distribution in large myelinated spinal root axons of the adult cat. J Neurocytol 22:925-940.

Calvo J, Funalot B, Ouvrier RA, Lazaro L, Toutain A, De Mas P, Bouche P, Gilbert-Dussardier B, Arne-Bes MC, Carrière JP, Journel H, Minot-Myhie MC, Guillou C, Ghorab K, Magy L, Sturtz F, Vallat JM, Magdelaine C (2009) Genotype-phenotype correlations in Charcot-Marie-Tooth disease type 2 caused by mitofusin 2 mutations. Arch Neurol 66:1511-1516.

Chen H, Chomyn A, Chan DC (2005) Disruption of fusion results in mitochondrial heterogeneity and dysfunction. J Biol Chem 280:26185-26192.

Cheng HC, Ulane CM, Burke RE (2010) Clinical progression in Parkinson disease and the neurobiology of axons. Ann Neurol 67:715-725.

de Brito OM, Scorrano L (2008) Mitofusin 2 tethers endoplasmic reticulum to mitochondria. Nature 456:605-610.

Detmer SA, Chan DC (2007) Complementation between mouse Mfn1 and Mfn2 protects mitochondrial fusion defects caused by CMT2A disease mutations. J Cell Biol 176:405-414.

Dziedzic T, Metz I, Dallenga T, König FB, Müller S, Stadelmann C, Brück W (2010) Wallerian degeneration: a major component of early axonal pathology in multiple sclerosis. Brain Pathol 20:976-985.

Fischer LR, Glass JD (2007) Axonal degeneration in motor neuron disease. Neurodegener Dis 4:431-442.

Hogan V, White K, Edgar J, McGill A, Karim S, McLaughlin M, Griffiths I, Turnbull D, Nichols P (2009) Increase in mitochondrial density within axons and supporting cells in response to demyelination in the Plp1 mouse model. J Neurosci Res 87:452-459.

Kang JS, Tian JH, Pan PY, Zald P, Li C, Deng C, Sheng ZH (2008) Docking of axonal mitochondria by syntaphilin controls their mobility and affects short-term facilitation. Cell 132:137-148.

Kim JY, Shen S, Dietz K, He Y, Howell O, Reynolds R, Casaccia P (2010) HDAC1 nuclear export induced by pathological conditions is essential for the onset of axonal damage. Nat Neurosci 13:180-189.

Li YC, Zhai XY, Ohsato K, Futamata H, Shimada O, Atsumi S (2004) Mitochondrial accumulation in the distal part of the initial segment of chicken spinal motoneurons. Brain Res 1026:235-243.

Macaskill AF, Rinholm JE, Twelvetrees AE, Arancibia-Carcamo IL, Muir J, Fransson A, Aspenstrom P, Attwell D, Kittler JT (2009) Mirol is a calcium sensor for glutamate receptor-dependent localization of mitochondria at synapses. Neuron 61:541-555.

Malek SA, Adorante JS, Stys PK (2005) Differential effects of Na-K-ATPase pump inhibition, chemical anoxia, and glycolytic blockade on membrane potential of rat optic nerve. Brain Res 1037:171-179.

Miller KE, Sheetz MP (2004) Axonal mitochondrial transport and potential are correlated. J Cell Sci 117:2791-2804.

Mironov SL (2007) ADP regulates movements of mitochondria in neurons. Biophys J 92:2944-2952.

Misgeld T, Kerschensteiner M, Bareyre FM, Burgess RW, Lichtman JW (2007) Imaging axonal transport of mitochondria in vivo. Nat Methods 4:559-561.

Misko A, Jiang S, Wegorzewska I, Milbrandt J, Baloh RH (2010) Mitofusin 2 is necessary for transport of axonal mitochondria and interacts with the Miro/Milton complex. J Neurosci 30:4232-4240.

Morfini GA, Burns M, Binder LI, Kanaan NM, LaPointe N, Bosco DA, Brown RH Jr, Brown H, Tiwari A, Hayward L, Edgar J, Nave KA, Garberrn J, 
Atagi Y, Song Y, Pigino G, Brady ST (2009) Axonal transport defects in neurodegenerative diseases. J Neurosci 29:12776-12786.

Murchison D, Griffith WH (2007) Calcium buffering systems and calcium signaling in aged rat basal forebrain neurons. Aging Cell 6:297-305.

Saotome M, Safiulina D, Szabadkai G, Das S, Fransson A, Aspenstrom P, Rizzuto R, Hajnóczky G (2008) Bidirectional $\mathrm{Ca}^{2+}$-dependent control of mitochondrial dynamics by the Miro GTPase. Proc Natl Acad Sci U S A 105:20728-20733.

Stirling DP, Stys PK (2010) Mechanisms of axonal injury: internodal nanocomplexes and calcium deregulation. Trends Mol Med 16:160-170.

Stys PK, Ransom BR, Waxman SG, Davis PK (1990) Role of extracellular calcium in anoxic injury of mammalian central white matter. Proc Natl Acad Sci U S A 87:4212-4216.

Stys PK, Waxman SG, Ransom BR (1992) Ionic mechanisms of anoxic injury in mammalian CNS white matter: role of $\mathrm{Na}+$ channels and $\mathrm{Na}(+)-$ $\mathrm{Ca} 2+$ exchanger. J Neurosci 12:430-439.

Trapp BD, Nave KA (2008) Multiple sclerosis: an immune or neurodegenerative disorder? Annu Rev Neurosci 31:247-269.

Trapp BD, Stys PK (2009) Virtual hypoxia and chronic necrosis of demyelinated axons in multiple sclerosis. Lancet Neurol 8:280-291.

Twig G, Elorza A, Molina AJ, Mohamed H, Wikstrom JD, Walzer G, Stiles L, Haigh SE, Katz S, Las G, Alroy J, Wu M, Py BF, Yuan J, Deeney JT, Corkey BE, Shirihai OS (2008) Fission and selective fusion govern mitochondrial segregation and elimination by autophagy. EMBO J 27:433-446.

Vallat JM, Ouvrier RA, Pollard JD, Magdelaine C, Zhu D, Nicholson GA, Grew S, Ryan MM, Funalot B (2008) Histopathological findings in hereditary motor and sensory neuropathy of axonal type with onset in early childhood associated with mitofusin 2 mutations. J Neuropathol Exp Neurol 67:1097-1102.

Verburg J, Hollenbeck PJ (2008) Mitochondrial membrane potential in ax- ons increases with local nerve growth factor or semaphorin signaling. J Neurosci 28:8306-8315.

Verhoeven K, Claeys KG, Züchner S, Schröder JM, Weis J, Ceuterick C, Jordanova A, Nelis E, De Vriendt E, Van Hul M, Seeman P, Mazanec R, Saifi GM, Szigeti K, Mancias P, Butler IJ, Kochanski A, Ryniewicz B, De Bleecker J, Van den Bergh P, et al. (2006) MFN2 mutation distribution and genotype/phenotype correlation in Charcot-Marie-Tooth type 2. Brain 129:2093-2102.

Vickers JC, Dickson TC, Adlard PA, Saunders HL, King CE, McCormack G (2000) The cause of neuronal degeneration in Alzheimer's disease. Prog Neurobiol 60:139-165.

Vincent AM, Edwards JL, McLean LL, Hong Y, Cerri F, Lopez I, Quattrini A, Feldman EL (2010) Mitochondrial biogenesis and fission in axons in cell culture and animal models of diabetic neuropathy. Acta Neuropathol 120:477-489.

Wang X, Schwarz TL (2009) The mechanism of $\mathrm{Ca}^{2+}$-dependent regulation of kinesin-mediated mitochondrial motility. Cell 136:163-174.

Witte ME, Bø L, Rodenburg RJ, Belien JA, Musters R, Hazes T, Wintjes LT, Smeitink JA, Geurts JJ, De Vries HE, van der Valk P, van Horssen J (2009) Enhanced number and activity of mitochondria in multiple sclerosis lesions. J Pathol 219:193-204.

Wolf JA, Stys PK, Lusardi T, Meaney D, Smith DH (2001) Traumatic axonal injury induces calcium influx modulated by tetrodotoxin-sensitive sodium channels. J Neurosci 21:1923-1930.

Züchner S, Mersiyanova IV, Muglia M, Bissar-Tadmouri N, Rochelle J, Dadali EL, Zappia M, Nelis E, Patitucci A, Senderek J, Parman Y, Evgrafov O, Jonghe PD, Takahashi Y, Tsuji S, Pericak-Vance MA, Quattrone A, Battaloglu E, Polyakov AV, Timmerman V, et al. (2004) Mutations in the mitochondrial GTPase mitofusin 2 cause Charcot-Marie-Tooth neuropathy type 2A. Nat Genet 36:449-451. 